\title{
The role of surgery in high grade neuroendocrine tumours of the lung
}

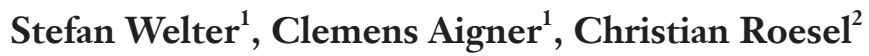 \\ ${ }^{1}$ Department of Thoracic Surgery and Thoracic Endoscopy, Ruhrlandklinik, West German Lunge Center, University Hospital, University of \\ Duisburg-Essen, 45239 Essen, Germany; ${ }^{2}$ Department of Thoracic Surgery, Lung Cancer Center, Bethanien Hospital Moers, Moers, Germany \\ Contributions: (I) Conception and design: All authors; (II) Administrative support: S Welter; (III) Provision of study materials or patients: S Welter, C \\ Roesel; (IV) Collection and assembly of data: C Roesel, S Welter; (V) Data analysis and interpretation: S Welter, C Roesel; (VI) Manuscript writing: \\ All authors; (VII) Final approval of manuscript: All authors. \\ Correspondence to: Stefan Welter, MD. Department of Thoracic Surgery and Thoracic Endoscopy, Ruhrlandklinik, West German Lunge \\ Center, University Hospital, University of Duisburg-Essen, Tueschener Weg 40, 45239 Essen, Germany. Email: stefan.we@t-online.de; \\ stefan.welter@ruhrlandklinik.uk-essen.de.
}

\begin{abstract}
High grade lung neuroendocrine tumours are a heterogeneous subtype of pulmonary cancers including small cell lung cancer (SCLC) and large-cell neuroendocrine carcinoma (LCNEC). LCNEC represents approximately $2-3 \%$ of lung cancers, whereas SCLC represents $15-20 \%$ of lung cancers. Patients with SCLC and LCNEC have a poor prognosis compared with patients with non-small cell lung cancer (NSCLC). LCNEC is treated with primary surgical resection in stages I-II, which is similar to other NSCLCs. Neo-adjuvant treatment in stage III is similar to NSCLC but has not been well studied. LCNEC tumours have an unfavourable prognosis in higher stages but a more favourable prognosis in earlier stages. Surgery plays a minor role in treatment of SCLC because tumours are often locally advanced or have metastasized at the time of presentation and treatment relies on chemo- or chemoradiotherapy. However, patients with limited cancer may demonstrate better disease control upon surgical treatment. The resection rate of limited disease (LD) SCLC is low (1-6\%), but 5-year survival rates of 31-42\% after surgical resection are encouraging and are significantly higher than the survival rates of comparable patients that did not have surgery. Curing SCLC in stage I is reported in up to $66 \%$ of cases. Local treatment with either resection or radiotherapy alone is followed by high rates of locoregional and distant recurrences, so preoperative or adjuvant treatment is recommended. Here, we summarise the similarities and differences of SCLC and LCNEC and highlight the role of surgery in the treatment of SCLC and LCNEC and its effect on local recurrence prevention.
\end{abstract}

Keywords: Lung cancer; neuroendocrine tumors of the lung; operation of small cell lung cancer (operation of SCLC); locoregional recurrence

Submitted Oct 17, 2016. Accepted for publication Dec 28, 2016.

doi: $10.21037 /$ jtd.2017.01.60

View this article at: http://dx.doi.org/10.21037/jtd.2017.01.60

\section{Introduction}

According to the World Health Organization (WHO) more than 1.5 million people are affected by lung cancer each year (1) from which one fifth are high grade lung neuroendocrine tumours. Pulmonary neuroendocrine tumours (NET) encompass four different groups: typical carcinoid (TC), atypical carcinoid (AC), large-cell neuroendocrine carcinoma (LCNEC) and small cell lung cancer (SCLC). They all have the potential to cause paraneoplastic syndromes and they may all express similar neuroendocrine markers. These neuroendocrine entities are further classified into two groups according to their biological aggressiveness: well-differentiated low grade (G1) TC and (G2) AC, and poorly-differentiated high grade (G3-4) LCNEC and (G4) SCLC $(2,3)$. Compared with carcinoid tumours, SCLC and LCNEC have much higher mitotic rates, more necrosis, and can present in combination with other lung cancer types including adenocarcinoma or squamous cell carcinoma (3). High grade NETs of the 

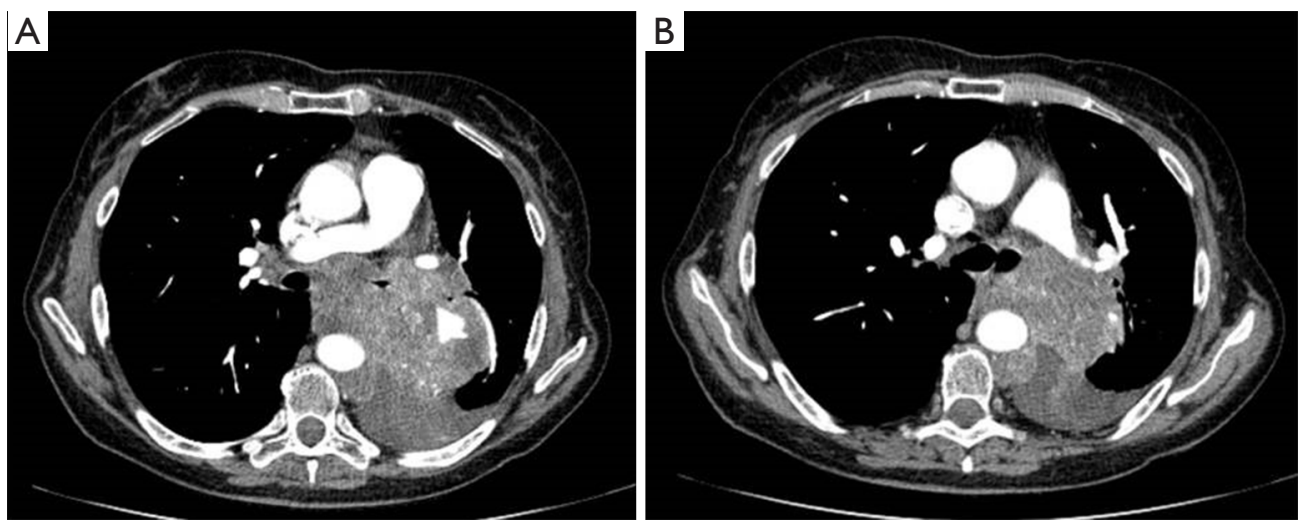

Figure 1 Centrally located small cell lung cancer (SCLC). Inoperable 68-year-old female, complaining about chest pain and shortness of breath. Heavy smoker for over 45 years. T4 tumour invading the pulmonary artery, left main bronchus, left atrium and subcarinal as well as contralateral hilar lymph nodes.

lung share some clinical features for example, they all begin to disseminate early in the course of the disease, patients are older than TC or AC patients, and nearly all of them are heavy cigarette smokers $(2,4,5)$. Clinical outcomes of patients with LCNEC are reported to be similar to SCLC, with 5-year survival rates of $15-57 \%(6-8)$. Localized LCNECs are currently treated like other nonsmall cell carcinomas $(8,9)$, but disease management is under debate. SCLC treatment relies on chemotherapy and chemoradiation therapy as most tumours have metastasized at the time of first diagnosis and only $4 \%$ of patients present with a solitary nodule. The rate of surgical resection for SCLC is $1-6 \%(10,11)$ and its role is not clearly defined. Since most studies and large case series are focused either on LCNEC or SCLC, we describe both types sequentially and aim to extract common features.

\section{SCLC}

SCLC accounts for $15-20 \%$ of all lung cancers and 34,000 new cases are expected each year in the United States. The clinical onset of symptoms is rapid and caused by central and bulky lesions (Figure 1), vena cava superior (VCS) compression or by early dissemination to regional lymph nodes and distant sites $(12,13)$. Approximately $10 \%$ of SCLC is associated with paraneoplastic syndromes, such as syndrome of inappropriate antidiuretic hormone secretion (SIADH), Cushing syndrome, Lambert-Eaton syndrome, and hypercalcemia (13).

Only $10-20 \%$ of the patients initially present with early stage disease and can be treated with curative intent. Due to early dissemination, chemotherapy is the most important treatment and the initial response to combination chemotherapy can be as high as $80-100 \%$. With the addition of thoracic irradiation, the 5 -year survival of patients with early stage SCLC can be increased from 5\% to $20 \%$ (14) and median survival can be increased from 9.9 to 17.7 months (15). Despite attempts to increase intensity of chemotherapy and radiation this type of tumour is associated with a high recurrence rate, at both local and distant sites. Local failure accounts for $30-50 \%$ of recurrences (16).

This paper aims to highlight the role of surgery in the treatment of SCLC and its effect on local recurrence prevention.

\section{Staging of SCLC}

The exact staging at the time of treatment initiation is of special importance for SCLC due to its tendency to undergo early, distant spread. Traditionally SCLC was classified into limited disease (LD) and extensive disease (ED). LD was characterized by tumours that are confined to one hemithorax, although local extension and ipsilateral supraclavicular nodes could also be present if they were located in the same radiation portal as the primary tumour. All other disease was classified as ED (12). Older studies that investigated the value of surgery, including surgery on LD-SCLC patients, found many inoperable cases (17). Therefore, a major issue in LD-SCLC is the risk of underestimating the local disease stage. This requires thorough preoperative investigation with modern imaging such as contrast enhanced spiral computed tomography (CT) scanning, whole brain MRI, and positron emission 
tomography (PET)-CT scanning completed by tissue examination of mediastinal nodes prior to resection (9). More recently clinicians recommend using the TNM staging system for SCLC, which may better subclassify LD and assist prognostication and guide future management (12). Candidates accepted for primary resection are in stage I (T1-2, N0, M0).

\section{Randomised trials to evaluate surgery for the treatment of SCLC}

To our knowledge there are only two randomised controlled trials that examine the role of surgery in the treatment of LD-SCLC. These are the "medical research council (MRC) comparative trial of surgery and radiotherapy for primary treatment of SCLC" (17) and the "prospective randomized trial to determine the benefit of surgical resection of residual disease following response of SCLC to combination chemotherapy" (18). Neither study found any survival benefit of tumour resection, but both studies had important limitations.

First, the MRC-UK randomised study showed a mean survival of 199 days for the surgery group and 300 days for the radical-radiotherapy group, which was a statistically significant difference $(\mathrm{P}=0.04)$. The main limitation of the study was that CT scanning and mediastinoscopy was not available for staging and thus most of the recruited patients were not candidates for surgery. Fifty-two percent of all patients underwent incomplete resection or exploratory thoracotomy due to unexpected locally advanced and inoperable disease (17). For a long time, these results reinforced existing reservations against surgery for SCLC and have been widely cited as evidence that surgical treatment of SCLC patients is ineffective.

The second study from Lad et al. (18) randomised 146 patients who achieved an objective response to cyclophosphamide, doxorubicin, and vincristine chemotherapy. All patients had central biopsy-proven SCLC. All biopsies were taken by rigid bronchoscopy from visible tumours. Seventy patients were randomly selected to receive surgical treatment and 76 were randomly selected to receive no surgical treatment. All randomised patients received radiotherapy to the chest and brain. Complete resection was achieved in $83 \%$ of patients and $19 \%$ of patients had pathologic complete remission. The median survival was 16 months for patients who were randomised. The survival curves for the two groups were not different $(\mathrm{P}=0.78)$ and the actuarial 2-year survival was $20 \%$. The authors, therefore, presumed that the results of this trial did not support the addition of pulmonary resection to the multimodality treatment of SCLC. The main limitations of this trial were the use of an older and less effective chemotherapy-regimen, the inclusion of only central tumours, and the fact that postoperative radiation therapy (PORT) included the whole former tumour area and that $35(7 \%)$ of the resected patients had positive lymph nodes.

\section{Primary resection for $S C L C$}

Primary resection is state of the art in stage I and II non-small cell lung cancer (NSCLC) (9). The question is whether primary resection is adequate for SCLC as well. Primary resection was evaluated only in a small series and with a different focus. Primary resection was performed with radical lymph node dissection in cases without conclusive preoperative SCLC histology (19), intentionally as primary treatment (20) and to evaluate adjuvant cisplatin/ etoposide chemotherapy (21). A median survival of 20 months (19) and a 5 -year survival of $11.1-52 \%$ were found (19-21). Adjuvant treatment was given in only $27.1 \%$ of 59 patients in the series outlined in Lim et al. (20) and the overall and disease free survival at 5 years was $52 \%$ and $46 \%$, respectively. In the other series with a high number of patients with adjuvant treatment 5 -year survival rates of $11-24 \%$ (19) and $56-66 \%$ (21) was found. The prognosis was poor for those with lobectomy compared to pneumonectomy cases $(\mathrm{P}=0.04)(19)$ and stage IIIa disease compared to stage I and II disease $(\mathrm{P}=0.02)(21)$.

\section{Mode of resection}

In modern thoracic surgeries pneumonectomy is avoided in lung cancer resection with bronchoplasty and vascular reconstruction techniques whenever possible and complete resection can be achieved. SCLC is typically located centrally (Figure 1) and, therefore, pneumonectomy and sleeve resections are more common. The pneumonectomy rate is $8.2-44 \%(14,19-22)$. A significantly $(\mathrm{P}=0.002)$ increased rate of local recurrence was found in the lobectomy group $(59.1 \%)$ versus the pneumonectomy group (22.6\%). This difference was found in stage I and II, but not in stage III disease (19). Others found much lower rates of local failure of $10 \%$ compared to distant relapses of $34 \%$ in a series of 62 resected patients (21). The radical resection of lymph nodes can lead to a high detection rate of unexpected node involvement. In two studies, $33 \%$ and $37 \%$ of patients 
Table 1 LD-SCLC treatment results from large cancer registries

\begin{tabular}{|c|c|c|c|c|c|}
\hline Author & SCLC cases & $\begin{array}{c}\text { Number of } \\
\text { R0-resections (\%) }\end{array}$ & $\begin{array}{l}\text { 5-year survival after } \\
\text { resection (\%) }\end{array}$ & $\begin{array}{l}\text { 5-year survival with } \\
\text { no surgery }(\%)\end{array}$ & Prognostic factors \\
\hline Vallières 2009 & 12,620 & $349(2.7)$ & $\begin{array}{l}48 \text { stage I; } 39 \text { stage } \\
\text { II; } 15 \text { stage III }\end{array}$ & - & N1 vs. N2 $(\mathrm{P}<0.0001)$ \\
\hline Schreiber 2010 & 14,179 & $863(6.1)$ & 34.6 & $9.9(P<0.001)$ & - \\
\hline Lüchtenborg 2014 & 45,282 & $465(1.0)$ & 31.0 & $3.1(P<0.001)$ & $\begin{array}{l}\text { PORT vs. no PORT in stage IIIA } \\
(P=0.01)\end{array}$ \\
\hline $\begin{array}{l}\text { Brock } 2005 \\
\text { (single centre) }\end{array}$ & 1,415 & $82(6.0)$ & 42.0 & - & $\begin{array}{l}\text { Lobectomy vs. limited resection } \\
\qquad(P=0.03)\end{array}$ \\
\hline
\end{tabular}

LD-SCLC, limited disease-small cell lung cancer; PORT, postoperative radiation therapy.

had clinical involvement of nodes and $48.1 \%$ and $63 \%$ had pathologic involvement in the final pathology after resection $(10,20)$.

\section{Primary radiotherapy}

In a randomised controlled trial, Turrisi et al. (16) treated patients with LD-SCLC. Cisplatin and etoposide were applied with twice-daily or once-daily thoracic radiotherapy up to 45 Gy and they explored local and distant failures. The rate was $52 \%$ in the group receiving once-daily therapy and $36 \%$ in the group receiving twice-daily therapy $(\mathrm{P}=0.06)$. The rates of simultaneous local and distant failure were significantly different between the groups. Both local and distant failure occurred in $23 \%$ of patients receiving once-daily therapy and $6 \%$ of patients receiving twicedaily therapy $(\mathrm{P}=0.01)$. This study highlighted that standard radiotherapy is insufficient to achieve local control and that local control even affects the rate of distant recurrences. And this study highlighted that intensification of local treatment increases rates of local and distant control.

The question of whether radiation therapy or surgery has a more important effect on local control was investigated by Zhang et al. (23). They reported approximately 153 SCLC patients with radical surgery $(n=50)$ and conservative $(\mathrm{n}=103)$ treatment. Both groups received chemotherapy. There were 85 patients out of 153 (55.6\%) that received thoracic radiotherapy, which was $74 \%$ of the non-surgical group and $20 \%$ of the surgical group. Despite the fact that most resected patients $(80 \%)$ had no radiation therapy, they found a significant difference $(\mathrm{P}=0.001)$ between the median survival rates in favour of the surgical group (30.5 months) versus the conservative group (16.9 months). Surgery was most advantageous in stage III (T4) patients $(\mathrm{P}=0.002)$.

\section{Surgical resection to improve LD-SCLC prognosis}

The proportion of resected LD-SCLC was very low in the past at $0-6.1 \%(11,16,22)$. Recently, large prospective cohort studies have demonstrated a potential benefit of resecting early stage SCLC (Table 1). A median overall survival benefit for surgical cases was demonstrated in localised (T1/T2) disease (42 vs. 15 months) as well as in regional (T3/T4) disease (22 vs. 12 months) with $\mathrm{P}<0.001$ for each type of disease (22). Limited resections are not recommended, as the prognosis is significantly $(\mathrm{P}=0.03)$ inferior to lobectomy (5-year survival $20 \%$ vs. $50 \%$ ) (24). The positive effects of complete resections were best shown with Kaplan-Meier survival curves of resected and unresected SCLC and NSCLC cases (Figure 2) (11).

\section{Resection in $\mathrm{N}+$ cases}

Stage IIIa SCLC cases are not recommended for surgery in any guideline. Nonetheless, complete resection of N2 cases can be beneficial with respect to survival in some series $(10,14,22)$. In a study by Schreiber et al. (22), a significant survival benefit was demonstrated in N0, N1 and N2 patients compared to non-surgery patients at the same stage (Table 2).

\section{Multimodality treatment}

Surgical treatment of SCLC is associated with high rates of local and distant failure $(14,19,21)$. Therefore, it seems reasonable to combine local and systemic treatment to increase long-term recurrence-free survival. This hypothesis was evaluated in a phase II study by Eberhardt et al. (14). Forty-six LD-SCLC patients in stages IB-IIIB 

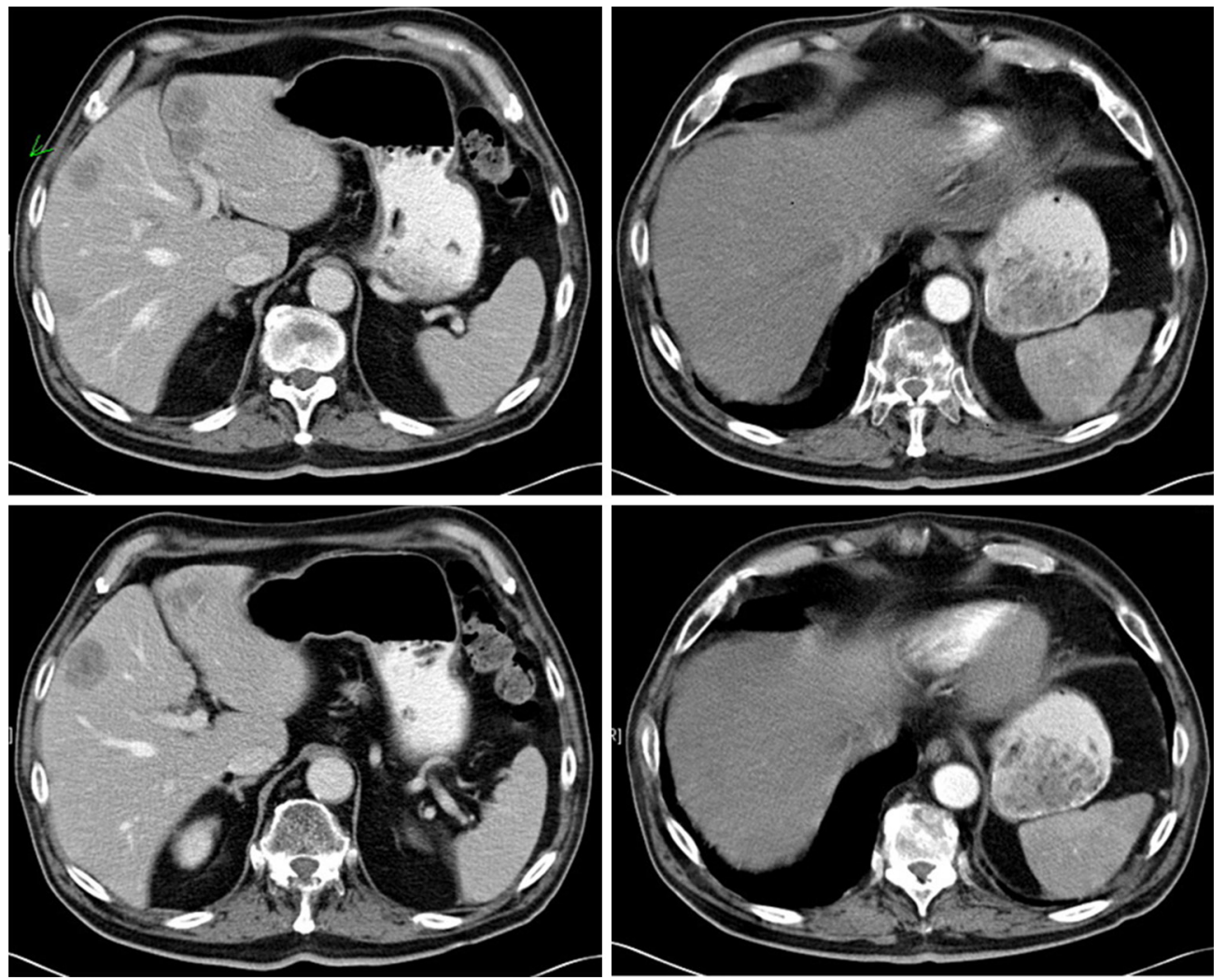

Figure 2 Distant recurrence of a stage IIa LCNEC after complete resection. 71-year-old male with distant recurrence 12 months after resection of a 5.2-cm LCNEC of the left upper lobe [pT2, pN1 (1/15), M0, R0, G3] followed by four cycles of adjuvant chemotherapy. Multiple liver metastases and one osteolytic lesion in the 10th thoracic vertebra were detected during regular follow-up.

Table 2 Effects of surgery by lymph node status retrieved from Schreiber D et al. [2010]

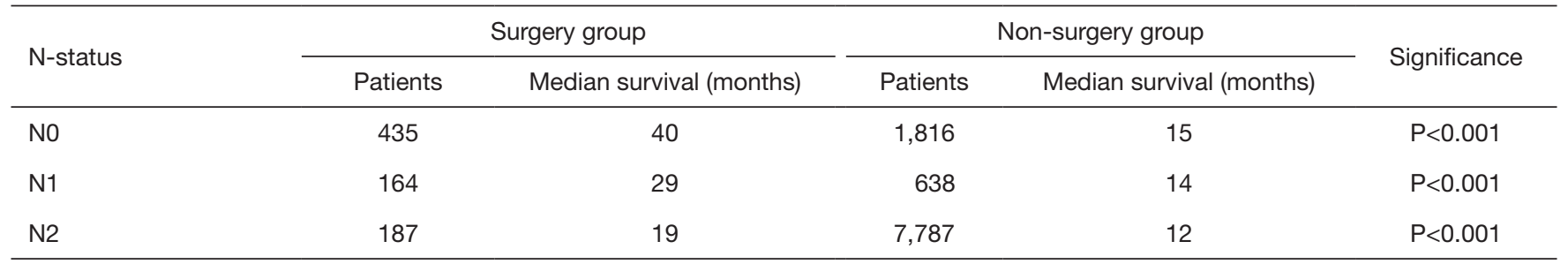

Data were retrieved from a SEER database that contains data from population-based national cancer registries in the US.

were selected and were treated with four cycles of cisplatin/ etoposide (PE) induction chemotherapy. Patients in stages IB-IIA underwent a tumour resection thereafter. Patients in stage IIB/IIIA had concurrent chemoradiotherapy and patients in stage IIIB received definitive chemoradiation treatment. There were 29 patients out of $46(63 \%)$ with proven mediastinal nodal disease. Repeat mediastinoscopy was performed after induction in all patients planned for surgery. Twenty-three of the planned 32 patients (72\%) were completely resected by 10 pneumonectomies, 2 bilobectomies and 11 lobectomies. The median OS was 36 months and the actuarial 5 -year survival was $46 \%$. Patients with complete resection had a median survival of 68 months and a 5 -year survival of $63 \%$. The non-surgery group had a significantly shorter median survival of 17 months and a 5 -year survival of $30 \%(\mathrm{P}=0.01)$. Tumour relapses 
occurred in the surgery and non-surgery group in 36\% and $61 \%$ of patients, respectively. After R0 resection, no locoregional recurrence was detected, whereas $26 \%$ of patients that did not undergo surgery had local failure. Thus, R0-resection seems to be an effective additional step to prevent locoregional recurrence in a multimodality setting.

\section{LCNEC}

The incidence of NETs is about $1.35 / 100,000 /$ year and has grown in recent decades. LCNEC accounts for $0.9 \%$ of all lung cancers and $40 \%$ of patients are in stage IV at initial diagnosis (25). The increasing detection rate is mainly the result of recent lung cancer screening programs and the improvement of available diagnostic tools $(26,27)$. Various series report that $85-98 \%$ of patients who had a surgical resection for LCNEC had a history of cigarette smoking. Therefore, smoking appears to be the primary cause for LCNEC. The mean age of patients treated for LCNEC ranged from $62-68$ years with a median of 65.8 years and patients with LCNEC were predominantly males (28-32). LCNEC frequently presents as a peripheral tumour in up to $85 \%$ of patients as opposed to TC and AC and SCLC, which are most often located centrally $(32,33)$. Histological differentiation between LCNEC and SCLC can be challenging because both tumour entities often share many common features, such as neuroendocrine morphology, large zones of necrosis, high mitotic rate, and positive immunohistochemical staining for neuroendocrine markers (34). The median OS of stages I-II, III and IV LCNEC patients was 32.4, 12.6 and 4.0 months, respectively, in a population-based cancer registry of the Netherlands. Survival is inferior to other NSCLC tumour types (25).

\section{Current standard of LCNEC treatment}

Due to the rarity of LCNEC, randomized clinical trials are difficult to perform and the therapeutic management of LCNEC is still controversial. The current NCCN treatment guideline recommends the same workup and treatment as other NSCLC tumours (9). Preoperative staging with PET-CT scanning, whole brain MRI, and contrast enhanced CT of the chest is necessary. Surgical resection is actually recommended for all non-metastatic stages, which is the same as the NSCLC treatment guideline, whereas adjuvant chemotherapy is predominantly administered, which is the same as the SCLC protocol
$(9,34)$. Primary operation is favoured in stages Ia to IIb, multimodality treatment is favoured for stages IIIa and IIIb, and palliative treatment is favoured in stage IV. Adjuvant chemotherapy is mostly administered as per SCLC protocols.

\section{Correct diagnosis}

As demonstrated in various publications, it is difficult to achieve an accurate diagnosis preoperatively $(35,36)$. In most cases, a conclusive pathological diagnosis was obtained from analysis of resection specimens.

\section{Outcome after surgery of LCNEC}

The clinical outcome of patients with LCNEC is reported to be similar to SCLC, with a 1-year survival rate of $27 \%$ (35) and a 5 -year survival rate of $13-57 \%(7,28)$. Radical surgery was reported with 5 -year survival rates varying from $10 \%$ to $88 \%(32,36,37)$. The prognosis after radical surgery is significantly inferior to other NSCLCs. This was demonstrated for pathologic stage IA tumours with 5-year survival rates of $54.5 \%$ for LCNEC compared to. $89.3 \%$ for NSCLC (37). The survival rates vary widely between different small retrospective series $(35,36)$. Larger operative series are quite similar, with 5 -year survival rates of $49.2 \%$ and $53.8 \%$ for LCNEC $(32,38)$. Stage I disease can be treated with higher success and survival rates of $64.5-88 \%$ $(32,36)$. In contrast, 5 -year survival after resection of stage I SCLC was $58 \%(24)$ and that of other NSCLCs was 66-88\% (39). Locally advanced (T3 and/or N2) LCNEC has a poor prognosis, showing a 1-year survival rate of only $27 \%$ after radical surgery and adjuvant chemotherapy, as reported in a small series of 18 patients (35). Thus, the prognosis of LCNEC patients is in between SCLC and NSCLC patients and is more like other NSCLCs in the early stages. However, many distant recurrences occur in LCNEC patients after complete resection (Figure 3). Therefore, the question arises if surgery alone is sufficient to treat LCNEC and if subsequent, more aggressive neoadjuvant or adjuvant chemotherapy concepts may be necessary $(40,41)$.

\section{Prognostic factors for LCNEC}

Large cell carcinomas with neuroendocrine features have a significantly inferior prognosis than other classic large cell lung cancers $(42,43)$. Immunohistochemical markers 


\begin{tabular}{|c|c|c|c|c|c|c|}
\hline \multicolumn{7}{|c|}{ NSCLC } \\
\hline "Limited disease" & \multicolumn{2}{c|}{ "Advanced disease" } \\
\hline N0 & N0 & N1 & N1 & N2 & N3 & Any \\
\hline IA & IB & IIA & IIB & IIIA & IIIB & IV \\
\hline \multicolumn{3}{|c|}{ Surgery } & \multicolumn{2}{c|}{ No routine surgery } \\
\hline
\end{tabular}

\begin{tabular}{|c|c|c|c|c|c|c|}
\hline \multicolumn{3}{|c|}{ SCLC } \\
\hline "limited disease" & \multicolumn{2}{|c|}{ "advanced disease" } \\
\hline N0 & N0 & N1 & N1 & N2 & N3 & Any \\
\hline IA & IB & IIA & IIB & IIIA & IIIB & IV \\
\hline \multicolumn{3}{|c|}{ Surgery } & \multicolumn{3}{|c|}{ No routine surgery } \\
\hline
\end{tabular}

Figure 3 Potential algorithm for the use of surgery in small cell lung cancer (SCLC) compared to non-small cell lung cancer (NSCLC). "Limited disease" (LD) means potentially operable from a surgeon's point of view and does not mean the old classification of SCLC restricted to one hemithorax accessible within one radiation field. The exclusion of viable N2-disease from surgery leaves the option to indicate resection for $\mathrm{N}$ - patients after induction chemoradiotherapy.

do not only allow differential diagnosis, but are also predictive. Coexpression of CD56 and CGA was associated with an increased rate of lymph node involvement and shorter survival. Staining for CD56, SYN-A, and CGA was positive in $86 \%, 81 \%$ and $61 \%$ of cases, respectively, in a recently published series (44). Roesel et al. (32) showed that lymphangitic spread, which can easily be detected by regular pathology, is a significant predictor of prognosis. Coexpression of neuroendocrine markers and lymphatic spread might be useful when deciding on adjuvant treatments. A gender difference concerning survival was only reported by Sarkaria $(45)$, but not by others $(32,44)$. Mediastinal lymph node involvement is a negative predictor, which was shown by a significant reduction in 5 -year survival between stages I and II $(52 \%, 59 \%)$ versus stage III $(20 \%, P=0.001)(7)$. Furthermore, an age of $>64$ years and the pT-stage were independent predictors of survival (38).

\section{Neo-adjuvant/adjuvant therapy}

Due to the small number of cases and the fact that all studies were retrospective, published discussions about adjuvant or neo-adjuvant chemotherapy regimens for LCNEC are still controversial.

LCNEC is most often responsive to platinum-based induction chemotherapy, with response rates of $60-80 \%$ $(7,45,46)$. Non-platinum chemotherapy is less effective, with response rates of $11 \%$ (46). Adjuvant platinum chemotherapy was shown to prolong survival $(37,45)$. The 5 -year survival rate following resection was $88 \%$ with adjuvant chemotherapy compared to $47 \%$ without adjuvant chemotherapy (37). Even in stage I LCNEC, the application of chemotherapy can improve outcome (7), but older studies did not uniformly report a survival improvement when platinum based adjuvant chemotherapy was used (28). Considering the supposed biological relation to SCLC and the comparable response rate, platin-based combination chemotherapy is generally recommended for advanced LCNEC (47), but may be beneficial for early stages as well.

\section{Discussion}

High grade NETs of the lung have a poor prognosis compared with other NSCLCs $(6-9,42,43)$. Whereas primary surgery is indicated in LCNEC tumours in stage $\mathrm{I} / \mathrm{II}$ and selected IIIa as in other NSCLCs, SCLC resection is recommended for stage I and non-N1 stage II. Often those tumours are resected without preoperative knowledge of high grad NET histology (19,32). Nevertheless, surgery alone does not seem to be sufficient to effectively treat high grade NETs $(7,19)$.

The value of surgery for the treatment of SCLC is still not exactly defined. Two randomised trials to evaluate the value of surgery for LD-SCLC failed to demonstrate any advantage $(17,18)$. Both trials, however, are old and did not include modern treatment and staging modalities, so they should not be used for current decision making. Nonetheless, these studies exhibited that LD-SCLC is often associated with a high rate of unexpected locally more advanced disease. This was verified by other studies later that showed a high rate of pathologically proven lymph nodes after resection $(10,14,20)$ and a high rate of necessary pneumonectomies $(14,19,20)$. Chemotherapy alone is also an insufficient treatment although there is a favourable response initially, followed by a local relapse rate of $90 \%$ after chemotherapy (48). The median survival for treatment of LD is $15-20$ months, with $20 \%$ surviving 2 years. For higher stage disease, the median survival is 
$8-13$ months with a 2 -year survival rate of $5 \%$ (13). These facts underline that even LD-SCLC treatment must always include systemic and local measures to prevent locoregional recurrence and to treat distant tumour cells. Frequent distant recurrence after resection is a well-known problem for LCNEC as well $(40,41)$ and consequently patients benefit from neoadjuvant or adjuvant chemotherapy plus radical resection $(7,37,45)$. The rate of local recurrence is lower for LCNEC than it is for SCLC because the tumours usually arise in the periphery and N2 involvement is less frequent. Local failure of resected SCLC after surgery alone or after surgery with chemotherapy is frequent and reduces survival $(19,20)$. Radiation therapy alone is also associated with a high rate of local failure $(14,16)$. The most promising results for stage II and IIIa patients (5-year survival of 63\%) were found for those selected candidates who had negative mediastinal nodes after pre-treatment with chemo- and chemoradiotherapy and then had radical surgery of the remaining primary tumour (14). A systematic Cochrane review evaluating the role of surgery in the treatment of SCLC is in progress (49).

Current treatment guidelines recommend platinumbased chemotherapy plus thoracic radiotherapy and prophylactic cranial irradiation (PCI) for the treatment of LD-SCLC $(5,9)$. The consideration of surgery is currently recommended for those who have a solitary nodule, no hilar or mediastinal involvement based on adequate mediastinal staging, no distant metastases, and no contraindications to surgery (9). Considering the positive influence of surgery on overall survival, even in N2-disease in several populationbased studies, we would encourage a wider indication of radical tumour resection in multimodality treatment including chemotherapy, radiotherapy and surgery for LD-SCLC. LCNEC should be treated as other NSCLCs but many authors recommend a more aggressive indication for adjuvant chemotherapy containing cisplatin or carboplatin and etoposide. Since distant recurrence is the main problem, we think that multimodality treatment concepts with neoadjuvant chemotherapy and surgery, as for stage II disease, should be implemented in the future. The role of PCI for LCNEC, as is used for treatment of SCLC, is still unclear and cannot be generally recommended $(9,50)$.

\section{Conclusions}

High grade lung NETs encompass LCNEC and SCLC. Both have a more aggressive clinical course than other NSCLCs with a high mitotic index and rapid growth.
LCNEC often present as peripheral tumours whereas SCLC typically arises as central masses. Both tumour types are found in older patients and heavy smokers. Response to platinum chemotherapy is around $60 \%$ for LCNEC and 80-90\% for SCLC, and metastases at initial diagnosis can be found in $40 \%$ for LCNEC and $60-80 \%$ for SCLC. The rate of primary resections is especially low at only $1-6 \%$ for LD-SCLC, but its value is currently underestimated. Oncologic resection of LD-SCLC can improve local control rates and thus increase the probability of longterm cure. Lobectomy is more effective in localized stage I disease, whereas pneumonectomy might be more effective in stage II-IIIA disease. Both tumour entities are associated with a high rate of local and distant recurrences, which implies that surgery or radiotherapy must be supported by pre- or postoperative chemotherapy in almost every stage and then followed by PCI for SCLCs.

We think that surgery can be beneficial in far more situations than named in the NCCN guideline. Wellselected N1 and N2 cases may benefit from multimodality treatment including surgery.

\section{Acknowledgements}

None.

\section{Footnote}

Conflicts of Interest: The authors have no conflicts of interest to declare.

\section{References}

1. Amini A, Byers LA, Welsh JW, et al. Progress in the management of limited-stage small cell lung cancer. Cancer 2014;120:790-8.

2. Fisseler-Eckhoff A, Demes M. Neuroendocrine tumours of the lung. Cancers 2012;4:777-98.

3. Travis WD, Brambilla E, Nicholson AG, et al. The 2015 World Health Organization classification of lung tumours: impact of genetic, clinical and radiologic advances since the 2004 classification. J Thorac Oncol 2015;10:1243-60.

4. Siegel R, Naishadham D, Jemal A. Cancer statistics, 2012. CA Cancer J Clin 2012;62:10-29.

5. Kalemkerian GP, Akerley W, Bogner P, et al. National Comprehensive Cancer Network. Small cell lung cancer. J Natl Compr Canc Netw 2013;11:78-98.

6. Asamura H, Kameya T, Matsuno Y, et al. Neuroendocrine 
neoplasms of the lung: a prognostic spectrum. J Clin Oncol 2006;24:70-6.

7. Veronesi G, Morandi U, Alloisio M, et al. Large cell neuroendocrine carcinoma of the lung: a retrospective analysis of 144 surgical cases. Lung Cancer 2006;53:111-5.

8. Varlotto JM, Medford-Davis LN, Recht A, et al. Should large cell neuroendocrine lung carcinoma be classified and treated as a small cell lung cancer or with other large cell carcinomas? J Thorac Oncol 2011;6:1050-8.

9. NCCN Clinical Practice Guidelines in Oncology (NCCN Guidelines $^{\circledR}$ ). Small cell lung cancer. Accessed October 8, 2016. Available online: https://www.nccn.org/ professionals/physician_gls/f_guidelines.asp\#sclc

10. Vallières E, Shepherd FA, Crowley J, et al. The IASLC Lung Cancer Staging Project: proposals regarding the relevance of TNM in the pathologic staging of small cell lung cancer in the forthcoming (seventh) edition of the TNM classification for lung cancer. J Thorac Oncol 2009;4:1049-59.

11. Lüchtenborg M, Riaz SP, Lim E, et al. Survival of patients with small cell lung cancer undergoing lung resection in England, 1998-2009. Thorax 2014;69:269-73.

12. Shepherd FA, Crowley J, Van Houtte P, et al. The International Association for the Study of Lung Cancer Lung Cancer Staging Project: proposals regarding the clinical staging of small cell lung cancer in the forthcoming (seventh) edition of the tumour, node, metastasis classification for lung cancer. J Thorac Oncol 2007;2:1067-77.

13. van Meerbeeck JP, Fennell DA, De Ruysscher DM. Smallcell lung cancer. Lancet 2011;378:1741-55.

14. Eberhardt W, Stamatis G, Stuschke M, et al. Prognostically orientated multimodality treatment including surgery for selected patients of small-cell lung cancer patients stages IB to IIIB: long-term results of a phase II trial. Br J Cancer 1999;81:1206-12.

15. Albain KS, Crowley JJ, LeBlanc M, et al. Determinants of improved outcome in small-cell lung cancer: an analysis of the 2,580-patient Southwest Oncology Group data base. J Clin Oncol 1990;8:1563-74.

16. Turrisi AT 3rd, Kim K, Blum R, et al. Twice-daily compared with once-daily thoracic radiotherapy in limited small-cell lung cancer treated concurrently with cisplatin and etoposide. N Engl J Med 1999;340:265-71.

17. Fox W, Scadding JG. Medical Research Council comparative trial of surgery and radiotherapy for primary treatment of small-celled or oat-celled carcinoma of bronchus. Ten-year follow-up. Lancet 1973;2:63-5.
18. Lad T, Piantadosi S, Thomas $\mathrm{P}$, et al. A prospective randomized trial to determine the benefit of surgical resection of residual disease following response of small cell lung cancer to combination chemotherapy. Chest 1994;106:320S-323S.

19. Jiang Y, Zhang Z, Xie C. Surgical resection for small cell lung cancer: pneumonectomy versus lobectomy. ISRN Surgery 2012;2012. doi:10.5402/2012/101024

20. Lim E, Belcher E, Yap YK, et al. The Role of Surgery in the Treatment of Limited Disease Small Cell Lung Cancer. Time to Reevaluate. J Thorac Oncol 2008;3:1267-71.

21. Tsuchiya R, Suzuki K, Ichinose Y, et al. Phase II trial of postoperative adjuvant cisplatin and etoposide in patients with completely resected stage I-IIIa small cell lung cancer: the Japan Clinical Oncology Lung Cancer Study Group Trial (JCOG9101). J Thorac Cardiovasc Surg 2005;129:977-83.

22. Schreiber D, Rineer J, Weedon J, et al. Survival outcomes with the use of surgery in limited-stage small cell lung cancer: should its role be re-evaluated? Cancer 2010;116:1350-7.

23. Zhang J, Li S, Chen X, et al. Retrospective study of surgery versus non-surgical management in limited-disease small cell lung cancer. Thorac Cancer 2014;5:405-10.

24. Brock MV, Hooker CM, Syphard JE, et al. Surgical resection of limited disease small cell lung cancer in the new era of platinum chemotherapy: its time has come. J Thorac Cardiovasc Surg 2005;129:64-72.

25. Derks JL, Hendriks LE, Buikhuisen WA, et al. Clinical features of large cell neuroendocrine carcinoma: a population-based overview. Eur Respir J 2016;47:615-24.

26. Yao JC, Hassan M, Phan A, et al. One hundred years after "carcinoid": epidemiology of and prognostic factors for neuroendocrine tumours in 35,825 cases in the United States. J Clin Oncol 2008;26:3063-72.

27. Oberg K, Hellman P, Kwekkeboom D, et al. Neuroendocrine bronchial and thymic tumours: ESMO clinical practice guidelines for diagnosis, treatment and follow-up. Ann Oncol 2010;21 Suppl 5:v220-2.

28. Dresler CM, Ritter JH, Patterson GA, et al. Clinicalpathologic analysis of 40 patients with large cell neuroendocrine carcinoma of the lung. Ann Thorac Surg 1997;63:180-5.

29. Takei H, Asamura H, Maeshima A, et al. Large cell neuroendocrine carcinoma of the lung: a clinicopathologic study of eighty-seven cases. J Thorac Cardiovasc Surg 2002;124:285-92.

30. Paci M, Cavazza A, Annessi V, et al. Large cell 
neuroendocrine carcinoma of the lung: a 10-year clinicopathologic retrospective study. Ann Thorac Surg 2004;77:1163-7.

31. Doddoli C, Barlesi F, Chetaille B, et al. Large cell neuroendocrine carcinoma of the lung: an aggressive disease potentially treatable with surgery. Ann Thorac Surg 2004;77:1168-72.

32. Roesel C, Terjung S, Weinreich G, et al. A singleinstitution analysis of the surgical management of pulmonary large cell neuroendocrine carcinomas. Ann Thorac Surg 2016;101:1909-14.

33. García-Yuste M, Matilla JM. The significance of histology: typical and atypical bronchial carcinoids. Thorac Surg Clin 2014;24:293-7.

34. Iyoda A, Makino T, Koezuka S, et al. Treatment options for patients with large cell neuroendocrine carcinoma of the lung. Gen Thorac Cardiovasc Surg 2014;62:351-6.

35. Mazières J, Daste G, Molinier L, et al. Large cell neuroendocrine carcinoma of the lung: pathological study and clinical outcome of 18 resected cases. Lung Cancer 2002;37:287-92.

36. Zacharias J, Nicholson AG, Ladas GP, et al. Large cell neuroendocrine carcinoma and large cell carcinomas with neuroendocrine morphology of the lung: prognosis after complete resection and systematic nodal dissection. Ann Thorac Surg 2003;75:348-52.

37. Iyoda A, H iroshima K, Moriya Y, et al. Prospective study of adjuvant chemotherapy for pulmonary large cell neuroendocrine carcinoma. Ann Thorac Surg 2006;82:1802-7.

38. Fournel L, Falcoz PE, Alifano M, et al. Surgical management of pulmonary large cell neuroendocrine carcinomas: a 10-year experience. Eur J Cardiothorac Surg 2013;43:111-4.

39. Goldstraw P, Crowley J, Chansky K, et al. The IASLC Lung Cancer Staging Project: proposals for the revision of the TNM stage groupings in the forthcoming (seventh) edition of the TNM Classification of malignant tumours. J Thorac Oncol 2007;2:706-14.

Cite this article as: Welter S, Aigner C, Roesel C. The role of surgery in high grade neuroendocrine tumours of the lung. J Thorac Dis 2017;9(Suppl 15):S1474-S1483. doi: 10.21037/ jtd.2017.01.60
40. Fernandez FG, Battafarano RJ. Large-cell neuroendocrine carcinoma of the lung. Cancer Control 2006;13:270-5.

41. Kozuki T, Fujimoto N, Ueoka H, et al. Complexity in the treatment of pulmonary large cell neuroendocrine carcinoma. J Cancer Res Clin Oncol 2005;131:147-51.

42. Battafarano RJ, Fernandez FG, Ritter J, et al. Large cell neuroendocrine carcinoma: an aggressive form of non-small cell lung cancer. J Thorac Cardiovasc Surg 2005;130:166-72.

43. Iyoda A, Hiroshima K, Toyozaki T, et al. Clinical characterization of pulmonary large cell neuroendocrine carcinoma and large cell carcinoma with neuroendocrine morphology. Cancer 2001;91:1992-2000.

44. Eichhorn F, Dienemann H, Muley T, et al. Predictors of survival after operation among patients with large cell neuroendocrine carcinoma of the lung. Ann Thorac Surg 2015;99:983-9.

45. Sarkaria IS, Iyoda A, Roh MS, et al. Neoadjuvant and adjuvant chemotherapy in resected pulmonary large cell neuroendocrine carcinomas: a single institution experience. Ann Thorac Surg 2011;92:1180-6.

46. Sun JM, Ahn MJ, Ahn JS, et al. Chemotherapy for pulmonary large cell neuroendocrine carcinoma: similar to that for small cell lung cancer or non-small cell lung cancer? Lung Cancer 2012;77:365-70.

47. Iyoda A, Hiroshima K, Moriya Y, et al. Postoperative recurrence and the role of adjuvant chemotherapy in patients with pulmonary large-cell neuroendocrine carcinoma. J Thorac Cardiovasc Surg 2009;138:446-53.

48. Elias AD. Small cell lung cancer: state-of-the-art therapy in 1996. Chest 1997;112:251S-258S.

49. Barnes H, See K, Barnett S, et al. Surgery for localised small cell lung cancer. Cochrane Database Syst Rev 2015. doi: 10.1002/14651858.CD011917

50. Rieber J, Schmitt J, Warth A, et al. Outcome and prognostic factors of multimodal therapy for pulmonary large-cell neuroendocrine carcinomas. Eur J Med Res 2015;20:64. 